\title{
Magnetic Force Scanning Tunneling Microscope Imaging of Overwritten Data
}

\author{
R. D. Gomez, A. A. Adly, and I.D. Mayergoyz \\ Institute for Advanced Computer Studies, Department of Electrical Engineering \\ University of Maryland, College Park, Maryland 20742 \\ E.R. Burke \\ Laboratory for Physical Sciences, College Park, Maryland 20740
}

\begin{abstract}
The magnetic force scanning tunneling microscopy is briefly discussed and demonstrated to successfully image overwritten data on commercial rigid magnetic disks. In particular, clear images of unerased remnants of the previously stored data are presented. These unerased data are attributed to slight radial deviations in write head tracking due to the intrinsic limitations in the electromechanical positioning of the head.
\end{abstract}

\section{INTRODUCTION}

One of the most increasingly active areas in magnetic recording technology is the study of processes occurring at the microscopic level. In recent years, several experimental techniques based on scanning tunneling microscopy have been developed to study magnetization patterns in recording media with sub-micron resolution. These include magnetic force microscopy (MFM) [1,2], and tunneling stabilized (TS) [3] or magnetic force scanning tunneling microscopy (MFSTM) [4]. We have actively pursued investigations using the MFSTM, and we demonstrate its capability to detect localized residual magnetization patterns on rigid disks arising from incomplete erasure during data overwrite.

To make this paper self-contained, we first provide a brief description of the technique and review key factors affecting its resolution and image contrast. We then present images of the overwritten data on commercial disks, as well as, the images of deliberately written pattems which serve as benchmark. Finally, we discuss the usefulness of this method for probing the subtle effects of insufficient erasure during data overwrite.

\section{EXPERIMENTAL TECHNIQUE}

Fig.1 shows a schematic diagram of the technique. A flexible magnetic probe, held at some small bias potential is placed in close proximity to the sample surface such that electrons from the surface can tunnel across the gap (or vice versa). As the probe is rastered across the surface, its vertical position is continuously adjusted to keep the tunneling current constant (typically a few tenths of a nanoamp). The probe deflects as it interacts with the surface magnetic fields and consequently changes the tip-sample separation. The feedback compensates for this change and the amount

Manuscript received February 17, 1992. of the probe vertical displacement, $\Delta z$, is recorded as a function of its horizontal position. The image is thus formed as a two dimensional mapping of the variations in $z$ as a function of the lateral position, i.e., $\Delta z(x, y)$, which reflects both the surface topological and magnetic features. With the appropriate choice of probe properties, it is possible to substantially enhance the magnetic contribution over the topology.

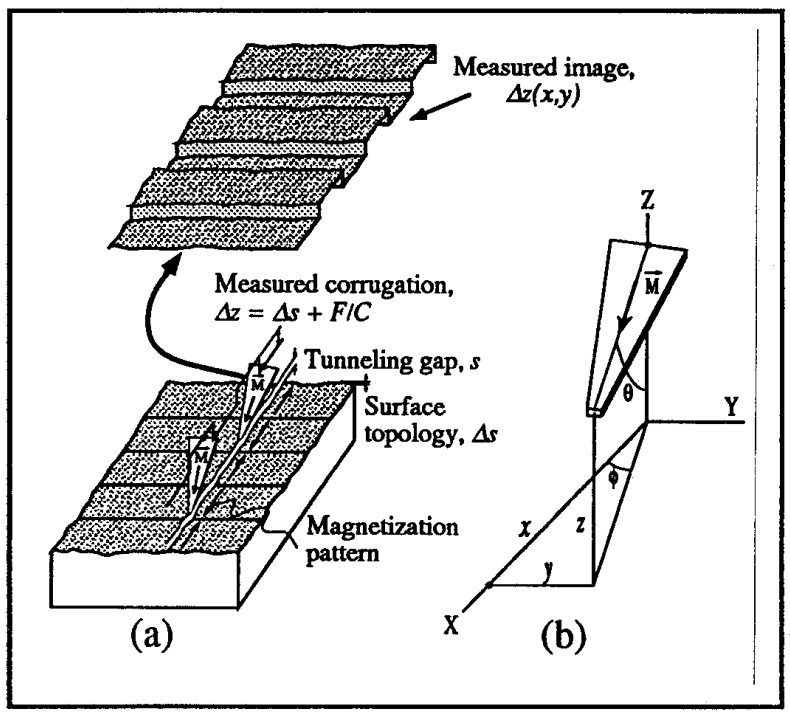

Fig. 1. Principle of operation of the magnetic force STM:

(a) schematic diagram, and (b) probe geometry.

The magnetic contribution to the displacement $\Delta \mathrm{z}$ is determined by the forces acting on the probe. There have been several theoretical calculations that relate the images with the forces on the probe $[2,6]$. However, these calculations have not directly addressed the issue of the dependence of image contrast and resolution on the orientation of the probe. In [4], the orientation dependence of the images have been specifically investigated. By assuming that the probe is uniformly magnetized along the direction of its length, it has been shown that the vertical displacement can be modeled by considering the interaction of the surface magnetic fields with a magnetic charge distribution at the apex of the tip $[4,5]$. Based on this, it has been shown that the variation in the tip-sample separation, $\Delta z$, due to magnetic interaction is governed by a simple expression, 


$$
\begin{gathered}
\Delta z=K\left[\sin (\theta) \cos (\theta) \cos (\phi) H_{x}+\sin ^{2}(\theta) H_{z}\right] \\
x \frac{\sin \left\{\frac{k w}{2} \sin (\phi)\right\}}{\frac{k w}{2} \sin (\phi)}
\end{gathered}
$$

where $K$ is some characteristic constant involving the probe mechanical and magnetic properties, $k$ is $2 \pi$ times the reciprocal of the pattern wavelength, $H_{x}$ and $H_{z}$ are the in-plane and out-of-plane components of the magnetic fields and the angles $(\theta, \phi)$ define the probe orientation relative to the surface magnetization. This relation explicitly shows the critical dependence of the image contrast on geometrical orientation of the probe. In addition, the observed magnetic corrugations represent a mixing of the in-plane $\left(H_{X}\right)$ and out-ofplane $\left(H_{z}\right)$ components of the field, with their relative weight contributions determined by the orientation. In the images obtained in our study, we have chosen the condition $\theta \sim \pi / 4$ and $\phi \sim 0$, to yield equal weights for $H_{x}$ and $H_{z}$.

Flexible magnetic probes made of $\mathrm{Ni}$ films can conveniently be used in this technique. The use of $\mathrm{Ni}$ film flexible magnetic tips for STM based MFM has been previously documented by Moreland and Rice in [7], where they reported that the film was "cut with scissors". In our work, the probes were fabricated by evaporating about $500 \mathrm{~nm}$ thick high purity $\mathrm{Ni}$ on prepatterned substrates. These films retained the shape of the substrate pattern when peeled and enabled us to accurately control their geometry. Typical dimensions of these triangular probes are about $4 \mathrm{~mm}$ and $1.5 \mathrm{~mm}$ on each side [4]. It has been observed that these probes produce consistent images of magnetization patterns.

The samples were taken from two different hard disks: (i) a conventional commercial removable computer rigid disk and (ii) an experimental calibration disk with data deliberately written on a precision spin stand system. On the commercial disk, sufficient computer disk access has been performed prior to removal from the drive, to ensure that a substantial area of the disk has been recorded over. On the calibration disk, sets of $1 \mathrm{Mhz}$ and $500 \mathrm{Khz}$ transitions were written alternately, with at least $25 \mu$ separating the individual tracks. Small pieces $(1 \mathrm{~cm} \times 2 \mathrm{~cm})$ were cut from both disks to fit microscope sample holder, and the carbon coating was removed by degreasing. The surfaces of these samples, as well as, the probe were sputter-coated with a few hundred angstroms of gold to reduce the adverse effects of oxide formation on tunneling experiments.

\section{RESULTS AND DISCUSSION}

\section{A. Commercial Disk}

Fig. 2(a) shows a $50 \mu \times 50 \mu$ image of the commercial rigid disk with overwritten data. New data appear as alternating bright protrusions and dark depres- sions representing oppositely magnetized regions along the tracks. Remnants of the previously recorded data appear as localized regions extending by a few microns from the upper track edge. During normal readback, a conventional head averages the signal over the track width and remnant magnetization contribute as edge noise. The sampling region is too broad, however, to detect these remnant magnetizations distinctly, and thus, old data cannot be retrieved separately. The area between the overlapping data patterns that appear somewhat blurred is known as the erase band. This band, characterized by reduced intensity magnetized regions is a well-known artifact of longitudinal recording $[1,8]$.

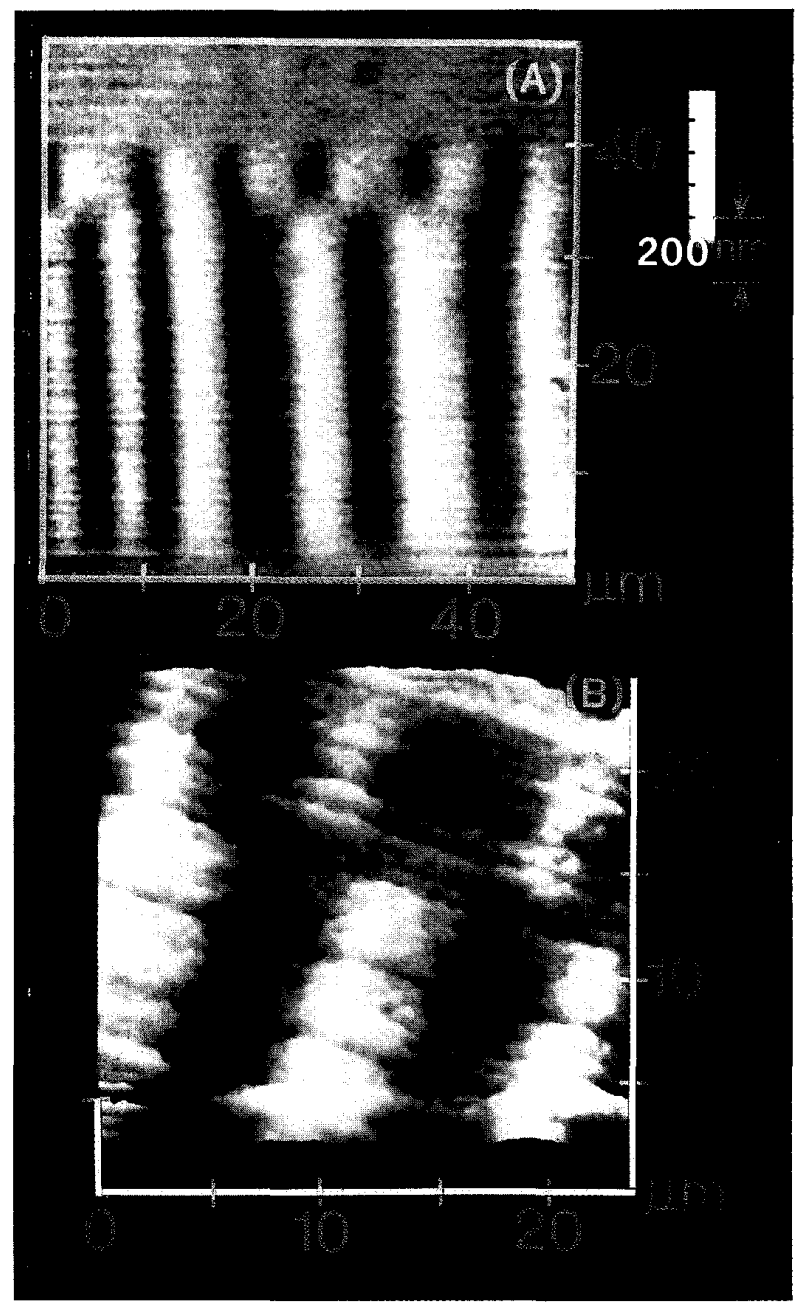

Fig.2. Overwritten data on conventional commercial rigid disk showing : (a) remnant of previous data, and (b) erase band.

The high resolution image of a different overwritten region in fig. 2(b) suggests some interesting characteristics of the erase band. The regions where the old and new data coincide create continuous magnetization between the old and new tracks, as exhibited by the ex- 
treme left transition. This is not the case for the two succeeding transitions, however, where the new set is out-of-phase with the old set. Here, the old data are truncated prior to the emergence of the new data, leaving about a micron wide gap with no definite magnetization. This behavior is consistent with current notions of the erase band $[1,8]$. The write field within this narrow band was above the coercivity of the media to reduce the magnetization at those areas (which truncated the bright stripes of the old data) but the magnitude was not high enough to create new well defined magnetizations.

The origins of the persistence of the old data can be inferred from the image as resulting from the slight radial off-track deviation of the head as the new set of data was recorded. In this specific case, the $5 \mu$ extension beyond the track edge is a measure of the ability of the servo-mechanism to maintain accurate positioning over the tracks. Thus, MFSTM investigations such as this can provide valuable information for evaluating the effectiveness of servo-positioning mechanisms, whose design requirements become increasingly stringent as track densities increase.

\section{B Calibration Disk}

A perspective-view MFSTM image of $500 \mathrm{KHz}$ and $1 \mathrm{MHz}$ transitions are shown in fig. 3 showing both topological and magnetic structures. These patterns were written by square wave signals with equal duration for positive and negative current polarities. The observed wavelengths of $40 \mu$ and $20 \mu$ are consistent with the $1 \mathrm{~F}$ and $2 \mathrm{~F}$ recording frequencies at the linear speed of $19 \mathrm{~m} / \mathrm{s}$. Note that for both signals the measured track width of about $25 \mu$ is substantially larger than the head pole width of about $14 \mu$. Close examination of the patterns in fig. 3 reveal bright flat regions at the centers of the tracks (indicated by arrows) that have widths very nearly equal to the width of the record

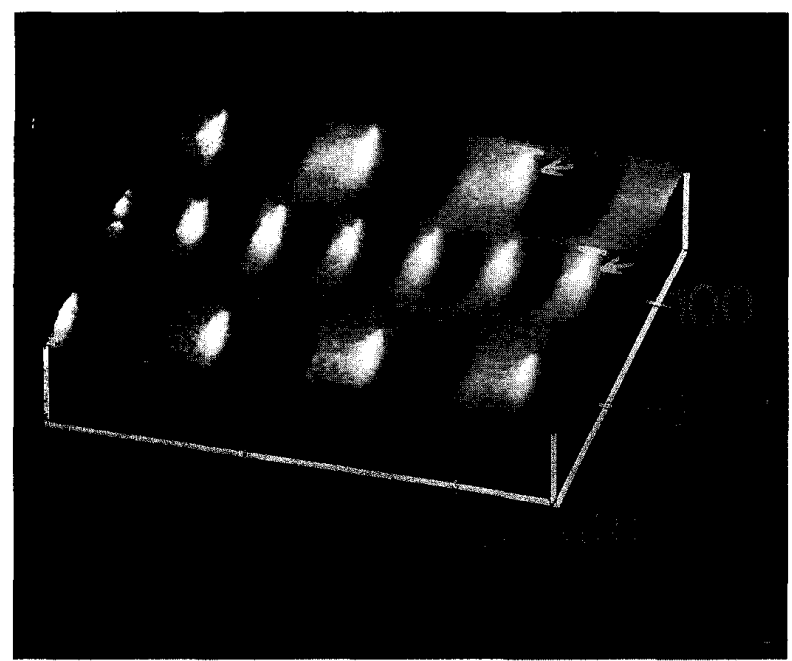

Fig. 3. Calibration disk. Perspective image of deliberately written $1 \mathrm{~F}(500 \mathrm{KHz})$ and $2 \mathrm{~F}(1 \mathrm{Mhz})$ magnetization patterns. head, and thus, the increase in the observed track width can be directly attributed to the fringing fields on both sides of the head.

It is clear that the efficiency of overwrite (erasure) will depend on fringing fields. As was shown in [8], fringing fields strongly depend on frequency, i.e., the fringing fields diminish with increasing frequency. Thus, the erase band width is similarly expected to diminish substantially at the higher recording frequencies. This effect will clearly play an important role in determining the minimum off-track deviation necessary before the remnant magnetization can persist. We have investigated this in detail, and our results will be presented elsewhere.

\section{CONCLUSION}

The MFSTM technique is shown to be a powerful technique for generating images of magnetization patterns. In particular, it has been used to yield images of persisting remnant data with minute details, and to investigate subtle features of overwritten data. Our present results demonstrate that due to the limitations in the electromechanical positioning of the recording head, overwritten data in longitudinal rigid disk recording systems is not necessarily completely erased. As a result, portions of the old data remain and could be retrieved. In the future, this technique can provide some valuable insights in various mechanisms associated with data overwrite, which include questions about the minimum off-track deviation necessary before the old data can persist.

\section{ACKNOWLEDGMENT}

We are grateful to Mr. John Gorczyca and Dr. Mark Kryder at Carnegie Mellon University, Data Storage Systems Center for their cooperation and for providing the $1 F / 2 F$ recorded rigid disks used in this experiment.

\section{REFERENCES}

[1] D. Rugar, H.J. Mamin, P. Guethner, S.E. Lamben, J.E. Stem, I. McFadyen and T. Yogi, "Magnetic force microscopy: general principles and application to longitudinal recording media", J. Appl. Phys., vol.68, p.1169, 1990.

[2] A.Wadas, P. Grütter and H.-J. Güntherodt," Analysis of in-plane bit structure by magnetic force microscopy", J. Appl. Phys., vol.67, p.3462, 1990 and references therein.

[3] P. Rice and J. Moreland, "Tunneling-stabilized Magnetic Force Microscopy of Bit Tracks on a Hard Disk", IEEE Trans. Magn., vol.27, p.3452, 1991

[4] R.D. Gomez, A.A. Adly, I.D. Mayergoyz and E.R. Burke, "Magnetic field imaging by using magnetic force scanning tunneling microscopy", Appl. Phys. Lett. Vol.60, p.906, 1992.

[5] E.R. Burke, R.D. Gomez, I.D. Mayergoyz and A.A.Adly, "Analysis of Tunneling Magnetic Force Microscopy Using a Flexible Triangular Probe", paper jb-09, Intermag Conf. 1992.

[6] J.J. Saenz, N. Garcia and J.C. Slonczewski, "Theory of magnetic imaging by force microscopy", Appl. Phys. Lett. 53, p.1449, 1988 and references therein.

[7] J. Moreland and P. Rice, "Tunneling stabilized, magnetic force microscopy with a gold-coated, nickel-film tip", J. Appl. Phys., vol.70, p.520, 1991 .

[8] H. Edelman and M. Covault,"Design of Magnetic Recording Heads for High Track Densities", IEEE Trans. Magnetics, vol .21, p.2583, 1985. 